\title{
Differential Impact of a Dutch Alcohol Prevention Program Targeting Adolescents and Parents Separately and Simultaneously: Low Self-Control and Lenient Parenting at Baseline Predict Effectiveness
}

\author{
Ina M. Koning • Jacqueline E. E. Verdurmen • \\ Rutger C. M. E. Engels • \\ Regina J. J. M. van den Eijnden • \\ Wilma A. M. Vollebergh \\ Published online: 2 February 2012 \\ (C) The Author(s) 2012. This article is published with open access at Springerlink.com
}

\begin{abstract}
To test whether baseline levels of the factors accountable for the impact of the Prevention of Alcohol use in Students (PAS) intervention (self-control, perceived rules about alcohol and parental attitudes about alcohol), moderate the effect of the intervention. A cluster randomized trial including 3,490 Dutch early adolescents $(M$ age $=$ 12.66, $S D=0.49$ ) and their parents randomized over four conditions: 1) parent intervention, 2) student intervention, 3) combined intervention and 4) control group. Moderators at baseline were used to examine the differential effects of the interventions on onset of (heavy) weekly drinking at 34month follow-up. The combined intervention was only effective in preventing weekly drinking among those adolescents who reported to have lower self-control and more lenient parents at baseline. No differential effect was found for the onset of heavy weekly drinking. No moderating roles of selfcontrol and lenient parenting were found for the separate student and parent interventions regarding the onset of
\end{abstract}

I. M. Koning $(\varangle) \cdot$ R. J. J. M. van den Eijnden ·

W. A. M. Vollebergh

Department of Interdisciplinary Social Science,

Utrecht University,

P.O. Box $80.140,3508$ TC Utrecht, the Netherlands

e-mail: i.koning@uu.nl

I. M. Koning $\cdot$ J. E. E. Verdurmen

Trimbos Institute,

Netherlands Institute of Mental Health and Addiction,

P.O. Box 725, 3500 AS Utrecht, the Netherlands

R. C. M. E. Engels

Behavioural Science Institute, Radboud University Nijmegen,

P.O. Box 9104, 6500 HE Nijmegen, the Netherlands drinking. The combined intervention is more effective among adolescents with low-self control and lenient parents at baseline, both factors that were a specific target of the intervention. The relevance of targeting self-control in adolescents and restrictive parenting is underlined.

Keywords Early adolescents · Alcohol use - Intervention . Randomized trial $\cdot$ Moderation

\section{Introduction}

In recent years, the number of Dutch adolescents drinking at a young age has increased (Monshouwer et al. 2008). At the same time more knowledge of the risks involved in underage drinking has become available (Behrendt et al. 2009; Brown et al. 2008). Although the literature shows mixed findings on the effectiveness of preventive alcohol interventions (Foxcroft et al. 2002; Spoth et al. 2008b), some interventions have proved to significantly affect the drinking behavior of adolescents. However, interventions may have different effects on different subgroups of adolescents. Thus, a relevant question is whether these effects apply to adolescents in general.

So far, most studies examining moderation effects focused on demographic moderators, such as gender, level of education and ethnicity, or on the initial level of outcome of interest (e.g., Brown et al. 2005; Sloboda et al. 2009; Spoth et al. 2006). For example, although most studies find more favorable effects of alcohol interventions in adolescents who drink more frequently at baseline (Brown et al. 2005; Spoth et al. 2008a), not much support for beneficial effects in other 
higher-risk groups (e.g., boys and adolescents in lower education) has been found (e.g., Spoth et al. 2006; Trudeau et al. 2003). However, it is particularly relevant to analyze whether the theoretically relevant factors that are targeted in the intervention also act as potential moderator variables.

Examination of the moderation effects of interventioninduced factors is important for two reasons. First, it provides theoretical evidence for the justification of targeting these specific factors in interventions. Second, insight into subgroups who have more favorable effects from an intervention helps in finding the best target groups for implementing this intervention (Kraemer et al. 2002), especially when these subgroups can be identified by demographic variables. Thus, it is relevant to examine the moderating role of the intervention-induced factors on the effect of the intervention condition on outcome of interest.

Recently, a Dutch school-based multi-component prevention program (PAS; Prevention of Alcohol use in Students) was shown to effectively postpone the onset of drinking by $10 \%$ when early adolescents as well as their parents were targeted (Koning et al. 2009), whereas targeting adolescents or parents separately revealed no significant effects. The adolescent part of the intervention was developed with a view to increasing self-control and fostering healthy attitudes in adolescents. This target behavior was chosen because the inability to refuse alcohol (low self-control) in adolescents appears to be a powerful predictor of (onset of) alcohol use in adolescents (e.g., Wills et al. 2001). Therefore, increasing the level of self-control in adolescents is often targeted in alcohol interventions, in many cases successfully (Foxcroft et al. 2002; Tobler et al. 2000). In the parent intervention, parents were encouraged to maintain restrictive rule setting and attitudes about alcohol use. This target behavior was chosen on the basis of a number of studies that consistently revealed strong effects of restrictive parenting (rules and attitudes about alcohol) on adolescent drinking (Koning et al. 2010b; Spijkerman et al. 2008; Van der Vorst et al. 2006, 2007; Yu 2003). That is, adolescents with stricter parents (e.g., who prohibit drinking) are less likely to drink alcohol (heavily). In addition, interventions targeting parenting behavior in order to induce change in their offspring's drinking have shown very promising results (Smit et al. 2008; Spoth et al. 2008b).

The combined intervention considered in this study revealed that this intervention indeed modified the theorybased factors as hypothesized. By increasing self-control among adolescents and by enhancing strict rule enforcement and negative attitudes in parents about adolescent alcohol use, the onset of weekly drinking was postponed (Koning et al. 2010a). Koning et al. (2009) showed that only when both adolescents and their parents were targeted was the onset of drinking postponed, whereas the separate parent or adolescent interventions were not effective. This makes us wonder if the combined intervention is differentially effective for different levels of self-control and restrictive parenting. In addition, the separate interventions may become effective if high levels of self-control in adolescents and perceived strict parenting are present at baseline. That is, the adolescent intervention may become effective when parents set restrictive rules and have strict attitudes, while the parent intervention may become effective among those adolescents with a high level of self-control.

According to the risk moderation hypothesis, an intervention should be more effective among high-risk groups than among moderate- and low-risk groups. Although this does not seem to account for moderation of demographic factors, it may apply to intervention-induced factors. Adolescents who lack the intervention-targeted protective behaviors can be considered among the high-risk groups. With respect to the PAS intervention, this involves adolescents with low self-control and low restrictive parents at baseline. Therefore, it can be expected that specifically those higher-risk groups of adolescents benefit more from the intervention.

As the student and parent interventions specifically target adolescent self-control and restrictive parenting respectively, more change can be induced in groups that are low at the start of the intervention in comparison to adolescents with high levels of these behaviors at baseline. Increasing selfcontrol in adolescents is often a target of alcohol interventions, in many cases successfully (Foxcroft et al. 2002; Tobler et al. 2000). Moreover, several studies point to the importance of testing self-control among adolescents as a moderator in intervention trials (e.g., Brown et al. 1998; Demmel et al. 2004). Earlier studies have confirmed that a lower level of self-control at baseline indeed appeared to be related to a higher degree of change in the desired direction during the intervention (Brown et al. 1998; Demmel et al. 2004). In addition, Brody et al. (2008) showed that the effect of their parenting program on conduct problems in adolescents was greater for youth with lower levels of self-control. Yet, to our knowledge, no data are available with respect to the moderation of restrictive parenting. Thus, a relevant question is to investigate whether the combined intervention is indeed most effective in the adolescent groups that are low in self-control and perceived rule setting at baseline and in parents with a tolerant attitude with respect to adolescent drinking.

\section{Current Study}

In this study, we examine whether the degree to which baseline levels of factors accountable for the effect of the combined PAS intervention (i.e., self-control, perceived parental rules and parental attitudes about alcohol use) moderate the effects of the different intervention conditions 
(parent and student intervention separately and simultaneously). In addition, in order to detect the subgroups on whom the intervention has more favorable effects, the characteristics of these subgroups have to be identified. Demographic factors that have been found to relate to low selfcontrol in adolescents or permissive parenting are gender (Sumter et al. 2009), age (Sumter et al. 2009; Van der Vorst et al. 2005), level of education (Spijkerman et al. 2008) and religion (Spijkerman et al. 2008). In a cluster randomized trial, the effects were measured for onset of both weekly and heavy weekly drinking at 34-month follow-up in a sample of 3,417 adolescents and their parents.

\section{Method}

Procedure and Participants

In April 2006, 80 schools were randomly selected from the list of all public secondary schools in the Netherlands, and were requested to participate in the study. A total of 20 schools were willing to participate.

Both the students and their parents were involved in this study. Student data were collected in classrooms by online questionnaires, available from a secure website. Research assistants were trained to administer the survey. Questionnaires for parents were sent to their home address, along with a letter of consent. Non-responding parents were encouraged to participate by a written reminder and phone call. The parent who completed the first questionnaire was asked to complete the subsequent questionnaires as well. In most cases (83\%), the responding parent was female. Both parental and student data were gathered in September/October 2006, before any intervention was carried out, and again 34 months later in June/July 2009. For a more detailed description of the procedure, see Koning et al. (2009).

\section{Randomization}

The participating schools were randomly assigned by an independent statistician to one of the following conditions: 1) parent intervention, 2) student intervention, 3) both parent and student intervention (combined intervention), or 4) control condition consisting of the regular curriculum. Randomization was carried out centrally, using a blocked randomization scheme (block size 5) stratified by level of education, with the schools as the unit of randomization. Within each participating school, all first-year students participated in the intervention. After randomization, one school refused further participation for reasons unrelated to the study. This school was originally randomized to the control condition.
Interventions

Parent Intervention (PI) This intervention targets parental rules for their children's alcohol use. The intervention was modeled on a Swedish intervention, The Örebro Prevention program (for details, see Koutakis et al. 2008). The intervention was carried out at the first parents' meeting at the beginning of each school year (September/October 2006 and 2007), in which other school-related topics were also discussed. A brief presentation was given at the plenary meeting by a social worker who was trained by the National Institute of Mental Health and Addiction. Thereafter, parents of children from the same class got together to reach consensus on a shared set of rules about alcohol use. Three weeks later an information leaflet with a summary of the presentation and a report of the outcome of the class meeting was sent to parents' home addresses.

Student Intervention (SI) The SI is the renewed digital alcohol module of the Dutch prevention program 'The Healthy School and Drugs' (HSD). The alcohol module makes use of e-learning and is based on principles of the theory of planned behavior (Ajzen and Fishbein 1990) and social cognitive theory (Bandura 1996). In the interactive lessons students were provided with information about alcohol and the risks involved in drinking. Students were trained to increase their self-control and develop healthy attitudes towards alcohol use by means of exercises such as short movies, texts following questions, animations and a chat room to exchange opinions. Each lesson ends with a summary task, such as creating a flyer or poster. The teachers received training in advance on how they could guide students in completing the lessons. In addition, information was given about how teachers could track the students while carrying out the intervention. The teachers conducted the intervention (four lessons) in all first-year classes in March/ April 2007. A hard-copy booster session was provided by the teachers 1 year later in March/April 2008. This booster lesson on paper involved a repetition of the digital alcohol program. For example, propositions were put forward to encourage discussion and recall knowledge obtained from the digital program.

Control Condition (CC) Schools in the control condition were contracted not to start any alcohol-related interventions throughout the study period. However, as basic information about alcohol use is part of the standard curriculum in the Netherlands, which involves the previously supported message of learning to drink alcohol safely instead of prohibiting it, they were allowed to continue this practice. The interventions were implemented and supervised by the National Institute of Mental Health and Addiction. 
Demographic Variables

Age (in years), gender, level of education and religion were demographic variables taken into account as covariates and/ or predictors. Age was measured by subtracting date of response from the date of birth of the respondent. The level of education reflects the type of education the adolescent engaged in. In the Netherlands, from the first year of secondary school, the educational system consists of different types of secondary education ranging from pre-vocational to pre-university education. This variable was dichotomized into lower (pre-vocational and lower secondary education) versus higher (higher general secondary and pre-university education) level of education. Religion was measured by asking if the adolescent was brought up religiously ('Catholic,' 'Protestant,' 'Islamic,' 'Other, namely' and 'No religion'). In the current analyses, adolescents indicating 'other religion' were recoded as missing (3.6\%). Dummy variables were computed with 'no religion' as reference category.

\section{Outcome Measures}

The outcomes of interest for the proposed study were equal to the outcome measures used in the previous study in which the effectiveness of the intervention was described (Koning et al. 2009). In accordance with the registration of this study in the Trial register (NTR649), we were interested in the effect of the intervention on the incidence rate of onset of drinking. Therefore, dichotomous variables were computed.

The onset of weekly alcohol use was defined by a Quantity-Frequency measure (Engels and Knibbe 2000; Engels et al. 1999). To detect the incidence rate of onset of weekly drinking, the scale was recoded into $0=$ 'no weekly use' and 1 = 'weekly user,' if at least one glass of alcohol was consumed on a weekly basis. This was only calculated for those adolescents who indicated a monthly prevalence of drinking. Onset of heavy weekly drinking was measured by asking how many glasses of alcohol the student usually drank on a weekend day (Engels et al. 1999). Boys drinking five or more glasses and girls drinking four glasses or more per week were considered to be heavy drinkers. The scale was recoded into a dichotomous variable with $0=$ 'no heavy weekly drinking' and 1 = 'heavy weekly drinking.'

\section{Moderators}

Self-control and restrictive parenting factors (rules and attitudes about alcohol) were moderators investigated in this study. All moderators, except parental attitudes, were reported by the adolescent. Due to skewness of the data (see Table 1), all moderators were transformed into dummies ( $0 / 1$ variables) on the basis of median split and were used to compute interaction variables (cf. Spoth et al. 2006).

Self-control measures the ability to control responses, to interrupt undesired behavioral tendencies and refrain from acting on them. The measure is the shorter version of the original measure developed by Tangney et al. $(2004)$. It consists of 13 items (alpha $=.74)$ that were rated on a 5-point scale, ranging from 1 'not at all like me' to 5 'very much like me.' For example, "I have trouble saying no" and "I do certain things that are bad for me, if they are fun." Items were reversely scored, higher scores indicate more self-control. The sample was divided into $0=$ 'high
Table 1 Descriptive statistics of demographic and moderating variables and alcohol use

$S D$ standard deviation

\begin{tabular}{|c|c|c|c|c|}
\hline \multicolumn{5}{|l|}{$\%$} \\
\hline \multicolumn{5}{|l|}{ Demographic variables } \\
\hline \multicolumn{5}{|l|}{ Religion } \\
\hline No religion & 59.5 & & & \\
\hline Catholic & 22.5 & & & \\
\hline Protestant & 13.6 & & & \\
\hline Islamic & 4.4 & & & \\
\hline \multicolumn{5}{|l|}{ Gender } \\
\hline Boy & 51.0 & & & \\
\hline \multicolumn{5}{|l|}{ Level of education } \\
\hline Low & 40.1 & & & \\
\hline \multicolumn{5}{|l|}{ Alcohol use } \\
\hline Weekly drinkers & 53.8 & & & \\
\hline Heavy weekly drinkers & 21.2 & & & \\
\hline Moderating variables & Mean & $S D$ & Skewness & Kurtosis \\
\hline Self-control (1-5) & 3.65 & 0.50 & -0.28 & 0.15 \\
\hline Rules about alcohol (1-5) & 4.64 & 0.46 & -2.04 & 6.06 \\
\hline Attitude toward alcohol (1-5) & 4.59 & 0.50 & -2.48 & 10.77 \\
\hline
\end{tabular}


self-control' and 1 = 'low self-control' based on the median score.

Rules about alcohol use reflect the degree of rule-setting behavior by the parents, as experienced by the adolescent (Van der Vorst et al. 2005). Items included "I am allowed to have one glass of alcohol when my parents are at home," "I am allowed to drink several glasses of alcohol when my parents are not home" and "I am allowed to drink alcohol when I am at a party with my friends." It consisted of the mean of ten items (alpha $=.90)$ rated on a 5-point scale from 1 'never' to 5 'always' reversely scored; i.e., higher scores indicate more rule-setting behavior. The median split was used to distinguish strict parents from lenient parents (scored as ' 0 ' and ' 1 ' respectively), as perceived by the adolescent.

Attitudes about alcohol use measure the degree to which the parent finds it acceptable $(1=$ not at all acceptable to $5=$ very acceptable) for a 12/13-year old adolescent to drink alcohol in various situations (Brody et al. 1999; Van der Vorst et al. 2006). Originally seven items were listed; in this study we added one item (drink alcohol on a Saturday evening with parents; alpha $=.79$ ). Responses were dichotomized, so that parents with strict and lenient attitudes (scored as ' 0 ' and ' 1 ' respectively) could be distinguished.

\section{Statistical Analyses}

Data were analyzed in accordance with the intent-to-treat principle. All analyses were conducted with Stata/SE version 9.2. At follow-up, $13.8 \%$ of the data were missing, mainly due to students changing schools. Missing data were handled by regressing imputation as implemented in Stata while using rules and attitudes about alcohol, alcohol use by both the students and their parents at baseline and age, gender, and level of education (lower vs. higher secondary education) as predictors of outcome.

The randomization had resulted in a slightly uneven distribution across the active conditions compared with the control condition in terms of age, gender, and level of education. Therefore, the moderation analyses were conducted with these variables as covariates to control for any possible bias stemming from the imbalance.

The cluster effect (introduced in the data since students were 'nested' in classes) was handled data-analytically by getting robust variance-related estimates based on the firstorder Taylor-series linearization method, using Stata's procedures for design-based analyses. We corrected for the cluster effects at class level, as the interventions were carried out in classes. To test moderation effects, interaction variables were computed between the moderator and the intervention dummies (received vs. did not receive the intervention). Furthermore, multiple logistic regression was used to analyze the main and moderation effects. Post estimation linear combination calculations, as implemented in Stata, were used to be able to interpret the significant moderation effects.

\section{Participants}

A total of 3,490 students were asked to participate in the study. Of these, 122 students did not participate due to the refusal of their parents, or because they were absent from school the day the questionnaire was administered. This resulted in a response rate of $97 \%(N=3,368)$ at baseline. It was required to include only students who did not meet the criteria for weekly drinking at baseline, and were therefore 'at risk' of becoming manifest as new cases of (heavy) drinking at follow-up. Therefore we excluded 431 students $(12.7 \%)$ who already were weekly drinkers at baseline $(n=$ $306)$, or who responded inconsistently on the quantity and frequency items measuring weekly drinking $(n=125)$. This resulted in a total of 2,937 students and $81 \%$ of their parents eligible for analysis. Of this group, a total of 2,533 students $(86.2 \%)$ and $63.3 \%$ of their parents stayed in the program and completed the follow-up assessment after 34 months. Intention-to-treat analyses were based on 2,937 students not manifesting weekly drinking at baseline and 2,381 parents.

\section{Characteristics of the Sample at Baseline}

The total student sample had a mean age of $12.66(S D=$ 0.49 ), consisting of $51 \%$ boys, and $40 \%$ in lower secondary education (Table 1). Most students reported not having any religion $(59.5 \%)$. An extensive description of the sample at baseline for each condition can be found in Koning et al. (2009). Of all participating parents, most were female (81\%) and between the age of 35 and 49 (90\%). More than one third of the parents had secondary education (38\%), 34\% vocational education and $27 \%$ third-level education.

\section{Results}

\section{Onset of Weekly Drinking}

Tables 2, 3 and 4 depict the main and interaction effects of intervention conditions with self-control and rules and attitudes about alcohol use respectively on the onset of weekly drinking.

Self-control in adolescents moderated the effect of the combined intervention on the onset of weekly drinking $\left(O R_{\text {interaction }}=0.62 ; 95 \% C I 0.40-0.96, p=0.04\right)$. Post estimation calculations showed that the effect of the combined intervention on weekly drinking was significant among adolescents with low self-control $(O R=0.52 ; 95 \%$ CI 0.36-0.75, $p=0.00$ ), and not significant among adolescents with high 
Table 2 Interaction effects between intervention conditions and selfcontrol on the onset of weekly drinking while controlling for age, gender and level of education

\begin{tabular}{lll}
\hline & OR & $95 \% C I$ \\
\hline Main effects & & \\
Age & 1.33 & $1.13-1.55$ \\
Gender $(1=$ boy) & 0.93 & $0.78-1.11$ \\
Level of education $(1=$ low $)$ & 1.17 & $0.95-1.45$ \\
Parent intervention & 1.00 & $0.65-1.54$ \\
Student intervention & 1.02 & $0.69-1.51$ \\
Combined intervention & 0.84 & $0.56-1.27$ \\
Self-control (1 = low) & 1.77 & $1.30-2.43$ \\
Interaction effects & & \\
Parent intervention x self-control & 0.70 & $0.42-1.17$ \\
Student intervention x self-control & 0.69 & $0.46-1.05$ \\
Combined intervention x self-control & 0.62 & $0.40-0.96$ \\
\hline
\end{tabular}

OR Odds Ratio, $C I$ Confidence Interval

ORs in italic are statistically significant $(p<.05)$

self-control $(O R=0.84 ; 95 \%$ CI $0.56-1.27, p=0.42)$. Thus, the combined intervention is only effective in adolescents with low self-control and not in adolescents with high selfcontrol at baseline (Fig. 1). No moderation effects of the student and parent interventions separately were found.

Rules about alcohol moderated the effect of the combined intervention on the onset of weekly drinking $\left(O R_{\text {interaction }}=\right.$ 0.63 ; 95\% CI 0.41-0.98, $p=0.04)$. Post estimation calculations showed that the effect of the combined intervention on weekly drinking was significant among adolescents reporting that their parents had lenient rules about alcohol use

Table 3 Interaction effects between intervention conditions and rules about alcohol on the onset of weekly drinking while controlling for age, gender and level of education

\begin{tabular}{lll}
\hline & $O R$ & $95 \% C I$ \\
\hline Main effects & & \\
Age & 1.37 & $1.17-1.61$ \\
Gender (1 = boy) & 0.93 & $0.78-1.11$ \\
Level of education $(1=$ low $)$ & 1.20 & $0.97-1.48$ \\
Parent intervention & 1.00 & $0.65-1.54$ \\
Student intervention & 1.01 & $0.72-1.44$ \\
Combined intervention & 1.01 & $0.71-1.44$ \\
Rules about alcohol $(1=$ lenient $)$ & 2.50 & $1.79-3.48$ \\
Interaction effects & & \\
Parent intervention x rules & 0.67 & $0.44-1.01$ \\
Student intervention x rules & 0.72 & $0.46-1.13$ \\
Combined intervention x rules & 0.63 & $0.41-0.98$ \\
\hline
\end{tabular}

OR Odds Ratio, $C I$ Confidence Interval

ORs in italic are statistically significant $(p<.05)$
Table 4 Interaction effects between intervention conditions and attitude about alcohol on the onset of weekly drinking while controlling for age, gender and level of education

\begin{tabular}{lll}
\hline & OR & $95 \%$ CI \\
\hline Main effects & & \\
Age & 1.38 & $1.16-1.64$ \\
Gender (1 = boy) & 0.95 & $0.79-1.15$ \\
Level of education (1 = low) & 1.18 & $0.94-1.47$ \\
Parent intervention & 0.76 & $0.57-1.41$ \\
Student intervention & 0.74 & $0.59-1.34$ \\
Combined intervention & 0.57 & $0.52-1.19$ \\
Attitude toward alcohol (1 = lenient) & 1.20 & $0.56-1.23$ \\
Interaction effects & & \\
Parent intervention x attitude & 1.18 & $0.68-2.06$ \\
Student intervention $x$ attitude & 1.19 & $0.71-2.02$ \\
Combined intervention x attitude & 1.37 & $0.83-2.26$ \\
\hline
\end{tabular}

OR Odds Ratio, CI Confidence Interval

ORs in italic are statistically significant $(p<.05)$

$(O R=0.63 ; 95 \%$ CI $0.41-0.98, p=0.00)$, and not significant among adolescents reporting to have strict parents $(O R=$ $0.84 ; 95 \%$ CI $0.59-1.19, p=0.32$ ). Thus, the combined intervention is effective in adolescents with lenient parents and not in adolescents with strict parents regarding alcohol use (Fig. 2). No moderation effects of the student and parent interventions separately were found.

Parental attitudes did not moderate the effect of any of the intervention conditions on the onset of weekly drinking.

\section{Onset of Heavy Weekly Drinking}

In addition to the direct effect of the combined intervention on the onset of heavy weekly drinking, no moderation effects of any of the intervention conditions were found. Thus, the effects of the interventions on the onset of heavy weekly drinking did not differ according to the level of self-control, perceived rule-setting and parental attitudes at baseline.

Additional Analysis

In order to be able to detect those subgroups of adolescents that respond most favorably to the combined intervention (a low level of self-control and low restrictive rules about alcohol), we performed logistic regression analyses with level of education, age, gender and religion (religion vs. no religion) at baseline as predictors, and level of selfcontrol (low vs. high) and rules about alcohol (low vs. high) as measures of outcome while controlling for the cluster effect.

Being Islamic as opposed to having no religion reduced the chance of having low self-control $(O R=0.47 ; 95 \% C I$ 
Fig. 1 Percentages (unadjusted) of weekly drinkers with low and high self-control in the combined and control condition

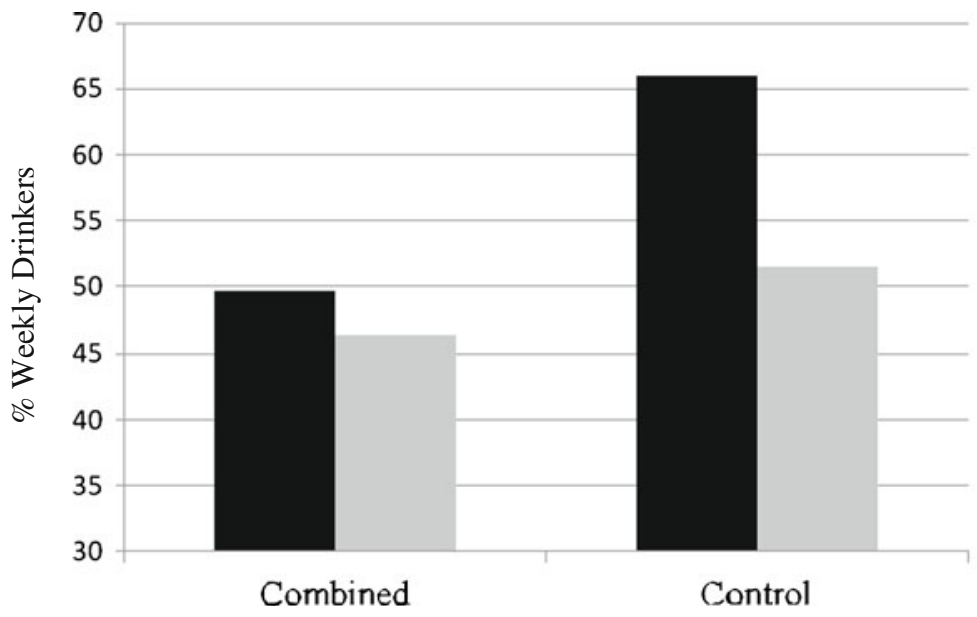

Low self-control

High self-control
$0.31-0.69, p=.000)$, while being at a lower level of education $(O R=1.99 ; 95 \% C I 1.64-2.43, p=.000)$ significantly increased the likelihood of having low self-control (Table 5).

Being Islamic had the only significant correlation with the level of rule setting in parents. Adolescents of Islamic religion (as opposed to no religion) have a lower risk of reporting low levels of restrictive parenting than adolescents reporting to have no religion $(O R=0.17 ; 95 \%$ CI $0.09-0.30$, $p=.000)$.

Logistic regression analysis performed on adolescents reporting both low self-control and low restrictive rule setting (25\% of the total sample) showed that, again, level of education and religion were significant correlates of having low self-control and low restrictive parents. That is, adolescents in low education $(O R=1.61 ; 95 \%$ CI $1.31-1.97, p=.000)$ are more likely and those of Islamic religion $(O R=0.16 ; 95 \% C I 0.08-0.35, p=.000)$ are less likely to have low self-control and to live with low restrictive parents.

\section{Discussion}

In the present study we examined the moderating effects of factors causing the effectiveness of an alcohol intervention targeting parents and adolescents simultaneously (PAS). Of all moderators investigated in this study (i.e., self-control, perceived rule-setting and parental attitudes about alcohol), both the initial level of self-control among adolescents and perceived rule-setting by parents moderated the effects of the combined intervention on the onset of weekly drinking. The present findings revealed that the combined intervention is most effective in delaying the onset of weekly drinking among those adolescents who reported to have low selfcontrol and lenient parents at baseline. This underscores the relevance of targeting self-control in adolescents and restrictive parenting in their parents. No differential effects of the separate parent and student intervention on delaying the onset of (heavy) weekly drinking were found.

The finding that adolescents who perceive their parents to be more lenient towards alcohol use and who experience
Fig. 2 Percentages (unadjusted) of weekly drinkers with lenient and strict parents in the combined and control condition

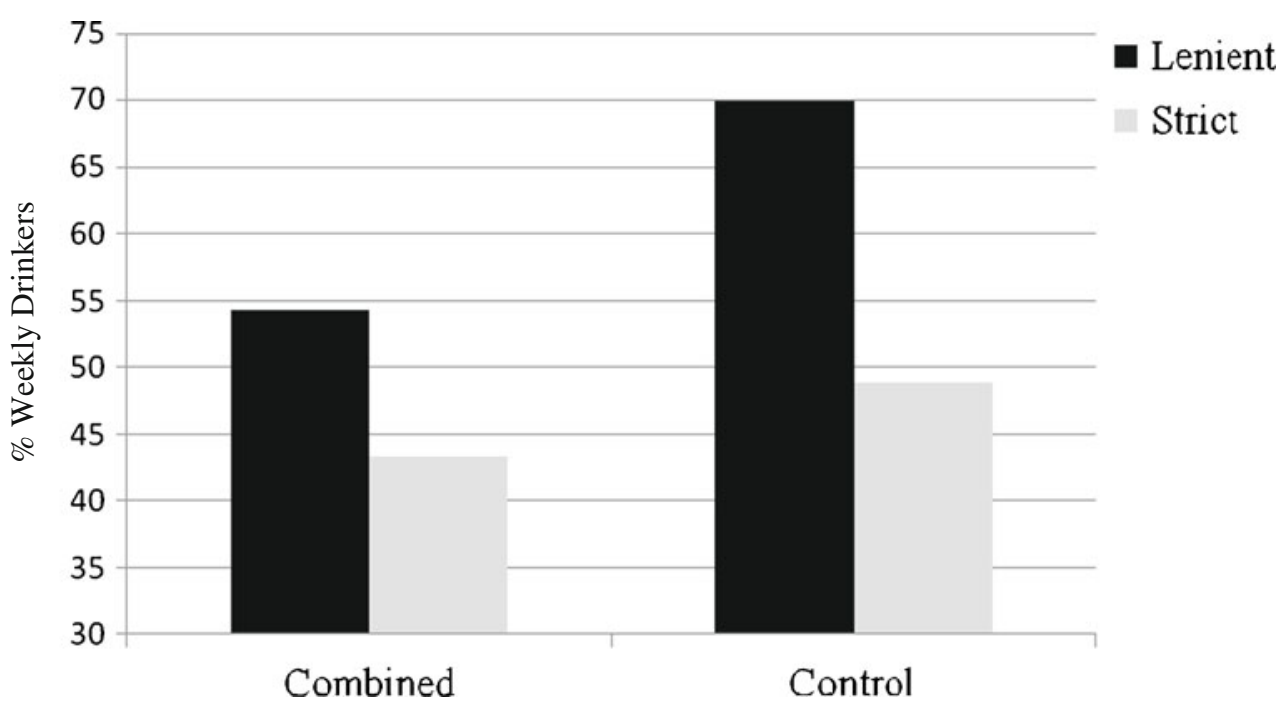


Table 5 Logistic regression of self-control and rules about alcohol predicted by demographic factors at baseline (gender, level of education and religion)

\begin{tabular}{|c|c|c|c|c|c|c|}
\hline & \multicolumn{2}{|c|}{ Self-control $(1=$ low $)$} & \multicolumn{2}{|c|}{ Rules about alcohol $(1=$ low $)$} & \multicolumn{2}{|c|}{ Self-control and rules about alcohol $(1=$ low on both $)$} \\
\hline & $O R$ & $p$-value & $O R$ & $p$-value & $O R$ & $p$-value \\
\hline Gender ( 1 = boy $)$ & 1.16 & .06 & 0.96 & .64 & 1.12 & .18 \\
\hline Age & 1.05 & .54 & 0.89 & .18 & 0.93 & .45 \\
\hline Level of education $(1=$ low $)$ & 1.99 & .00 & 1.15 & .14 & 1.61 & .00 \\
\hline \multicolumn{7}{|c|}{ Religion (reference $=$ no religion) } \\
\hline Catholic & 0.89 & .32 & 1.00 & .99 & 0.91 & .41 \\
\hline Protestant & .98 & .91 & 0.83 & .15 & 0.94 & .69 \\
\hline Islamic & .47 & .00 & 0.17 & .00 & 0.16 & .00 \\
\hline
\end{tabular}

OR Odds Ratio

ORs in italic are statistically significant $(p<.05)$

little behavioral control over their alcohol use benefit more from the combined intervention is in line with the risk moderation theory. According to the risk moderation hypothesis, high-risk groups should benefit more from preventive alcohol interventions than moderate- and low-risk groups. We defined high-risk groups in terms of the theoretically relevant target behaviors of the intervention; i.e., as those scoring low on the target behaviors at baseline. As PAS targeted self-control and restrictive parenting, adolescents with a low level of self-control and low restrictive parents were considered to be a high-risk group. As expected, lower levels of the behaviors targeted by PAS, resulted in more favorable effects of the intervention. The importance of the moderating role of self-control has already been demonstrated by Brown et al. (1998) in a sample of adults participating in an abstinence-focused inpatient alcohol treatment program. As far as we know, no studies are available that tested the moderating role of rules parents set about alcohol, that were also a target of the alcohol intervention. This study is one of the first showing the relevance of testing moderation of intervention-induced factors of alcohol interventions targeting early adolescents and their parents. In addition to the effects of PAS in the general population (Koning et al. 2009), the fact that the effects were more favorable among the groups scoring low on the intervention-induced behaviors at baseline underlines the appropriateness of choosing self-control and restrictive parenting as targets for the intervention.

We can only speculate about the lack of significance of moderation in respect of parental attitudes. Attitudes of parents about alcohol use, as opposed to specific rules about alcohol, represent not so much concrete behavior as a state of mind. It is possible that the moderating effect of the combined intervention on the onset of (heavy) weekly drinking was not found due to the, sometimes subconscious, cognitive acceptance of parents not to agree with early drinking. On the other hand, changing their behavior by setting rules requires more effort from parents and results in those parents who were lenient showing the highest rates of change.

The separate parent and student intervention parts did not become effective in subgroups of adolescents, based on their initial level of self-control and restrictive parenting. This may indicate that the effects of the separate interventions were not strong enough to foster change in onset of drinking even when the pertinent behaviors were low at baseline. However, the results do suggest that the separate interventions tend to be more beneficial for adolescents with low self-control and those experiencing lenient parenting. But in order to significantly impact the onset of drinking in early adolescents, behaviors in both parents and adolescents should be targeted. These findings underscore the additional effect of the combined intervention over and above the separate interventions.

While a differential effect of the combined intervention was found for the onset of weekly drinking, this was not the case for the onset of heavy weekly drinking. That is, the effect of the combined intervention on the onset of heavy weekly drinking did not differ across the level of selfcontrol and strict parenting. We can only speculate as to why the results are different for weekly and heavy weekly drinking. It is possible that self-control and parenting are mostly related to the onset of less severe drinking behavior, due to the high variance within adolescents and parents. In this way the intervention can induce high rates of change in a large number of adolescents scoring low on these behaviors. However, once adolescents drink heavily on a weekly basis, the lower variance of self-control and strictness of parents may explain the lack of differential effect in these subgroups. More research on the role of self-control and parenting in diverse drinking intensities could provide more insight into this issue.

Additional analyses showed that those at risk for having low self-control and low restrictive parents are particularly found 
among the lower educated and non-Islamic adolescents. This finding is in support of a previous study that demonstrated that adolescents from families with a lower socioeconomic status were more likely to have more lenient parents (Spijkerman et al. 2008). In addition, as drinking alcohol is not accepted in Islamic cultures and therefore alcohol use is not very common among immigrant groups (Monshouwer et al. 2003), parents of immigrant youth are expected to be more strict about alcohol. On the other hand, adolescents in lower education are at higher risk for alcohol use (Salonna et al. 2008). Thus, the level of education and religion of the adolescent are significant indicators for identifying the subgroups of adolescents who benefit most from the combined intervention.

\section{Study Limitations}

The results of this study should be considered in the light of some limitations. First, the results are based on self-reported questionnaires. Although susceptible to social desirability biases, self-reports on alcohol use are found to be a valid method to assess alcohol use in adolescents (Del Boca and Darkes 2003; Koning et al. 2010c). Second, the findings are based on one follow-up measurement only. Examining differential effects of the PAS intervention on alcohol use at later follow-ups may demonstrate the strength of the current results. Third, one should be careful in generalizing the findings to other countries with other drinking cultures. The Netherlands is considered to have a fairly lenient drinking culture, in which adolescents drink more frequently relative to other European countries (Hibell et al. 2009). Therefore, replication of this study in other countries is necessary in order to generalize these findings to countries with a stricter drinking culture. Fourth, although we managed to include the number of schools and participants that were needed to power the trial (see Koning et al. 2009), only $25 \%$ of all schools that were approached actually participated. No data are available on whether the participating schools differ from the non-participating schools. This may affect the generalizability of our findings. Last, in this study we included education, age, gender and religion as predictors to detect the specific subgroups that the intervention may benefit the most. It should be noted, however, that apart from these characteristics, other predictors could have been useful to include in the analyses, such as family situation and problematic behavior.

\section{Implications}

The present and previous results of the PAS intervention (Koning et al. 2009) indicate the need for targeting adolescents as well as parents, over and above targeting either of them separately in a school setting. It is suggested that the concurrent increase of self-control in adolescents and restrictive parenting in parents are behaviors that can be targeted effectively by alcohol interventions. Prevention workers and schools wanting to delay the onset of weekly drinking should be informed by the Netherlands Institute of Mental Health and Addiction about the importance of implementing the combined PAS intervention aimed at both students and their parents. Adolescents in lower levels of education and in non-Islamic school should be a specific target when implementing the PAS intervention. However, since no adverse effects were found in other groups of adolescents, PAS can be implemented in schools with adolescents from different religious and educational backgrounds. The present findings highlight the conclusion that the separate interventions should not be implemented. Even in cases where adolescents have high self-control and/ or parents have strict rules, the separate interventions are not shown to be effective, thereby indicating that an increase in these behaviors only is not sufficient to foster change. Prevention workers should be kept updated about new insights and knowledge that are obtained in the future.

Acknowledgements This study was funded by grant number 6220 0021 from the Dutch Health Care Research Organization (Z.O.N.-M. W). We would like to thank the Associate Editor and the three anonymous reviewers who provided us with thoughtful comments on the revision.

Conflict of interest The authors declare that there are no conflicts of interest.

Open Access This article is distributed under the terms of the Creative Commons Attribution Noncommercial License which permits any noncommercial use, distribution, and reproduction in any medium, provided the original author(s) and source are credited.

\section{References}

Ajzen, I., \& Fishbein, M. (1990). Understanding attitudes and predicting social behaviour. Englewood Cliffs, NJ: Prentice Hall.

Bandura, A. (1996). Social foundation of thought and actions: A social cognitive theory. Englewood Cliffs: Prentice Hall.

Behrendt, S., Wittchen, H. U., Höfler, M., Lieb, R., \& Beesdo, K. (2009). Transitions from first substance use to substance use disorders in adolescence: Is early onset associated with a rapid escalation? Drug and Alcohol Dependence, 99, 68-78.

Brody, G. H., Flor, D. L., Hollett-Wright, N., McCoy, J. K., \& Donovan, J. (1999). Parent-child relationships, child temperament profiles and children's alcohol use norms. Journal of Studies on Alcohol, 13, $45-51$.

Brody, G. H., Kogan, S. M., Yi-fu, C., \& McBride Murry, V. (2008). Long-term effects of the Strong African American Families Program on youths' conduct problems. Journal of Adolescent Health, 43, $474-481$.

Brown, S. A., Anderson, K. G., Schulte, M. T., Sintov, N. D., \& Frissell, K. C. (2005). Facilitating youth self-change through school-based intervention. Addictive Behaviors, 30, 1797-1810. 
Brown, S. A., Carello, P., Vik, P. W., \& Porter, R. J. (1998). Changes in alcohol and self-efficacy and alcohol expectancies during addiction intervention. Substance Abuse, 19, 155-167.

Brown, S. A., McGue, M., Maggs, J., Schulenberg, J., Hingson, R., Swartzwelder, S., et al. (2008). A developmental perspective on alcohol and youths 16 to 20 years of age. Pediatrics, 121, S290S310.

Del Boca, F. K., \& Darkes, J. (2003). The validity of self-reports of alcohol consumption: State of the science and challenges for research. Addiction, 98, 1-12.

Demmel, R., Beck, B., Richter, D., \& Reker, T. (2004). Readiness to change in a clinical sample of problem drinkers: Relation to alcohol use, self-efficacy, and treatment outcome. European Addiction Research, 10, 133-138.

Engels, R. C. M. E., \& Knibbe, R. A. (2000). Alcohol use and intimate relationships in adolescence: When love comes to town. Addictive Behaviors, 25, 435-439.

Engels, R. C. M. E., Knibbe, R. A., \& Drop, M. J. (1999). Why do late adolescents drink at home? A study on the psychological wellbeing, social integration and drinking context. Addiction Research, 7, 31-46.

Foxcroft, D.R., Ireland, D., Lowe, G., \& Breen, R. (2002). Primary prevention for alcohol misuse in young people. Cochrane Database of Systematic Reviews. 3, Art. No.: CD003024.

Hibell, B., Guttormsson, U., Ahlström, S., Balakireva, O., Bjarnason, T., Kokkevi, A., \& Kraus, L. (2009). The 2007 ESPAD report substance use among students in 35 European countries. Stockholm: The Swedish Council for Information on Alcohol and Other Drugs (CAN).

Koning, I. M., Engels, R. C. M. E., Verdurmen, J. E. E., \& Vollebergh, W. A. M. (2010). Alcohol-specific socialization practices and alcohol use in Dutch early adolescents. Journal of Adolescence, $33,93-100$.

Koning, I. M., Harakeh, Z., Engels, R. C. M. E., \& Vollebergh, W. A. M. (2010). A comparison of self-reported alcohol use measures by early adolescents: Questionnaires versus diary. Journal of Substance Use, 15, 166-173.

Koning, I. M., van den Eijnden, R. J. J. M., Engels, R. C. M. E., Verdurmen, J. E. E., \& Vollebergh, W. A. M. (2010). Why target early adolescents and parents in alcohol prevention? The mediating effects of self-control, rules and attitudes about alcohol use. Addiction, 106, 538-564.

Koning, I. M., Vollebergh, W. A. M., Smit, F., Verdurmen, J. E. E., van den Eijnden, R. J. J. M., ter Bogt, T. F. M., et al. (2009). Preventing heavy alcohol use in adolescents (PAS): Cluster randomized trial of a parent and student intervention offered separately and simultaneously. Addiction, 104, 16691678.

Koutakis, N., Stattin, H., \& Kerr, M. (2008). Reducing youth alcohol drinking through a parent-targeted intervention: The Örebro Prevention Program. Addiction, 103, 1629-1637.

Kraemer, H. C., Wilson, G. T., Fairburn, C. G., \& Agras, W. S. (2002). Mediators and moderators of treatment effects in randomized clinical trials. Archives of General Psychiatry, 59, 877-883.

Monshouwer, K., Smit, F., De Zwart, W. M., Spruit, I., \& Van Ameijden, E. J. C. (2003). Progress from a first drink to first intoxication: Age of onset, time-windows and risk factors in a Dutch national sample of secondary school students. Journal of Substance Use, 8, 155-163.

Monshouwer, K., Verdurmen, J., van Dorsselaer, S., Smit, E., Gorter, A., \& Vollebergh, W. (2008). Jeugd en riskant gedrag 2007
[Adolescents and risk-taking behaviour 2007]. Utrecht, The Netherlands: Trimbos-instituut.

Salonna, F., van Dijk, J.P., Geckova, A. M., Sleskova, M., Groothoff, J. W., \& Reijneveld, S. A. (2008). Social inequalities in changes in health-related behavior among Slovak adolescents aged between 15 and 19: A longitudinal study. BMC Public Health, 8.

Sloboda, Z., Stephens, R. C., Stephens, P. C., Grey, S. F., Teasdale, B., Hawthorne, R. D., et al. (2009). The adolescent substance abuse prevention study: A randomized field trial of a universal substance abuse prevention program. Drug and Alcohol Dependence, $102,1-10$

Smit, E., Verdurmen, J., Monshouwer, K., \& Smit, F. (2008). Family interventions and their effect on adolescent alcohol use in general populations: A meta-analysis of randomized controlled trials. Drug and Alcohol Dependence, 97, 195-206.

Spijkerman, R., van den Eijnden, R. J., \& Huiberts, A. (2008). Socioeconomic differences in alcohol-specific parenting practices and adolescents' drinking patterns. European Addiction Research, 14, 26-37.

Spoth, R., Greenberg, M., \& Turrisi, R. (2008). Preventive interventions addressing underage drinking: State of the evidence and steps toward public health impact. Pediatrics, 121, 311-336.

Spoth, R. L., Randall, G. K., Trudeau, L., Shin, C., \& Redmond, C. (2008). Substance use outcomes $5 \frac{1}{2}$ years past baseline for partnership-based family-school preventive interventions. Drug and Alcohol Dependence, 96, 57-68.

Spoth, R. L., Shin, C., Guyll, M., Redmond, C., \& Azevedo, K. (2006). Universality of effects: An examination of the comparability of long-term family intervention effects on substance use across riskrelated subgroups. Prevention Science, 7, 209-224.

Sumter, S. R., Bokhorst, C. L., Steinberg, L., \& Westenberg, P. M. (2009). The developmental pattern of resistance to peer influence in adolescence: Will the teenager ever be able to resist? Journal of Adolescence, 32, 1009-1021.

Tangney, J. P., Baumeister, R. F., \& Boone, A. L. (2004). High selfcontrol predicts good adjustment, less pathology, better grades, and interpersonal success. Journal of Personality, 72, 271-322.

Tobler, N. S., Roona, M. R., Ochshorn, P., Marshall, D. G., Streke, A. V., \& Stackpole, K. M. (2000). School-based adolescent drug prevention programs: 1998 meta-analysis. Journal of Primary Prevention, 20, 275-336.

Trudeau, L., Spoth, R., Lillehoj, C., Redmond, C., \& Wickrama, K. A. S. (2003). Effects of a preventive intervention on adolescent substance use initiation, expectancies, and refusal skills. Prevention Science, 4, 109-122.

Van der Vorst, H., Engels, R. C. M. E., Dekovic, M., Meeus, W., \& Vermulst, A. A. (2007). Alcohol-specific rules, personality and adolescents' alcohol use: A longitudinal person-environment study. Addiction, 102, 1064-1075.

Van der Vorst, H., Engels, R. C. M. E., Meeus, W., \& Dekovic, M. (2006). The impact of alcohol-specific rules, parental norms about early drinking and parental alcohol use on adolescents' drinking behavior. Journal of Child Psychology and Psychiatry, 47, 1299-1306.

Van der Vorst, H., Engels, R. C. M. E., Meeus, W., Dekovic, M., \& Van Leeuwe, J. (2005). The role of alcohol-specific socialization in adolescents' drinking behaviour. Addiction, 100, 1464-1476.

Wills, T. A., Cleary, S., Filer, M., Shinar, O., Mariani, J., \& Spera, K. (2001). Temperament related to early-onset substance use: Test of a developmental model. Prevention Science, 2, 145-163.

$\mathrm{Yu}$, J. (2003). The association between parental alcohol-related behaviors and children's drinking. Drug and Alcohol Dependence, 69, 253-262. 